\title{
Desarrollo profesional docente y educación a distancia. Una experiencia desde la cooperación sur-sur
}

\author{
Teacher Professional Development and Distance Education. An Experience \\ from South-South Cooperation
}

\author{
Marcela Peña-Ruz ${ }^{1}$
}

\begin{abstract}
RESUMEN
El siguiente artículo tiene como objetivo presentar el proyecto "Intercambio de experiencias pedagógicas entre El Salvador y Chile para fortalecer la formación ciudadana y la convivencia democrática", desarrollado por la Universidad de Chile, a través del Programa de Educación Continua para el Magisterio (PEC) ${ }^{2}$ de la Facultad de Filosofía y Humanidades, durante los años 2017, 2018 y 2019, en el marco de la política de cooperación Sur-Sur impulsada por el Gobierno de Chile mediante el Fondo Chile ${ }^{3}$. Se incluye un apartado de antecedentes generales, desarrollo de la experiencia y desafíos desde la educación continua para docentes y actores del sistema educativo.
\end{abstract}

Palabras claves: desarrollo profesional docente; formación de docentes; formación continua de docentes; educación a distancia para docentes; formación ciudadana; cooperación sur-sur.

\begin{abstract}
The following article aims to present the project "Exchange of Pedagogical Experiences Between El Salvador and Chile to Strengthen Citizen Education and Democratic Coexistence", developed by the University of Chile through the Continuing Education Program for Teaching (PEC) of the Faculty of Philosophy and Humanities, during the years 2017 to 2019, within the framework of the South-South cooperation policy promoted by the government of Chile through Chile Fund. The article includes a general background section, development of experience section and challenges from continuing education for teachers and actors in the educational system.
\end{abstract}

Keywords: Teacher professional development; teacher education; continuing teacher education; distance education for teachers; citizen education; South-South cooperation.

\footnotetext{
${ }^{1}$ Coordinadora del Núcleo de Estudio y Desarrollo "Liderazgo y Gestión Educativa", Centro Saberes Docentes, Universidad de Chile, Santiago, Chile; magíster en Educación; marcela.pena @uchile.cl.

${ }^{2}$ El año 2018 el PEC pasa a constituirse como Centro de Estudios y Desarrollo de Educación Continua para el Magisterio, alias Saberes Docentes.

${ }^{3}$ Este proyecto ha sido financiado por Fondo Chile, iniciativa del Gobierno de Chile, a través del Ministerio de Relaciones Exteriores y la Agencia Chilena de Cooperación Internacional para el Desarrollo (AGCID), en alianza con el Programa de las Naciones Unidas para el Desarrollo (PNUD).
} 


\section{Antecedentes del proyecto}

\subsection{Encuadre conceptual}

La comprensión de la formación continua de profesores y profesoras supone una concepción del desarrollo profesional docente como un proceso continuo y permanente en la escuela, que implica "volverse consciente de las propias prácticas pedagógicas, cuestionar sus visiones y preconcepciones hegemónicas y obtener retroalimentación y apoyo dirigido y específico" (Laveault, 2016, p.138) ${ }^{4}$. De este modo, la formación continua debe aportar al desarrollo profesional docente con modelos de formación que propicien el fortalecimiento de prácticas de reflexión pedagógica en contexto.

En América Latina la formación docente presenta una serie de características que en algunos casos se traducen en problemas, tales como "intensa actividad y bajo impacto; escasa relevancia y calidad de la oferta; desvinculación con la realidad; y desconocimiento de la realidad de docentes principiantes" (Vaillant, 2016, p 10).

Laveault (2016) plantea que, para que el desarrollo profesional de los y las docentes se traduzca en prácticas innovadoras en el aula, debe existir un modelo de regulación definido por tres dimensiones: la existencia de metas claras para los y las profesionales; la reflexividad y autoregulación; las acciones remediales y la toma de decisiones de los/as docentes.

De este modo, procesos de formación continua que promuevan el aprender desde la experiencia, la construcción de conocimiento colectivo con foco en el aprendizaje de estudiantes, el fortalecimiento de la investigación y el trabajo colaborativo, generan en forma articulada desarrollo profesional docente (Korthagen, Loughran y Russell, 2006).

Hoy el acceso a internet, uso de redes sociales y plataformas a través de teléfonos celulares y tablets, ha incrementado la oferta y demanda de formación en modalidad e-learning y blended learning (presencial y a distancia) (García-Ruiz, Aguaded y Bartolomé, 2017), siendo un desafío en estas modalidades la incorporación de metodologías que promuevan el desarrollo profesional docente.

\footnotetext{
${ }^{4}$ Traducción propia.
} 
DESARROLLO PROFESIONAL DOCENTE Y EDUCACIÓN A DISTANCIA. UNA EXPERIENCIA DESDE LA COOPERACIÓN SUR-SUR / PEÑA-RUZ

Duarte, Guzmán y Domínguez (2018), a partir de una revisión bibliográfica de artículos científicos sobre la educación a distancia para docentes, plantean que el blended learning es una modalidad óptima para el desarrollo profesional docente, que logra movilizar los aprendizajes docentes hacia el desarrollo de nuevas prácticas, a partir de diseños que articulen en forma sistémica lo virtual y lo presencial. Asimismo, señalan que es fundamental promover en las actividades de formación la colaboración entre pares y contar con el apoyo de las instituciones en que se desempeñan los y las docentes con el fin de potenciar su aprendizaje, tanto en los tiempos de dedicación como en las posibles innovaciones a integrar en los contextos educativos.

\subsection{Contextos nacionales en formación ciudadana}

El Salvador y Chile en los últimos años han desarrollado diversas líneas políticas para el fortalecimiento de la formación ciudadana en educación, a través de un conjunto de leyes que desafían a los centros educativos a plantearse estas temáticas en forma consistente en sus proyectos educativos.

- En Chile durante el año 2016 se promulga la Ley 20.911 que compromete a los establecimientos escolares a incluir en todos los niveles de enseñanza un Plan de Formación Ciudadana, en forma transversal y sistémica, de modo que esta temática se incorpora tanto en aspectos pedagógicos como de gestión en cada centro educativo. Este desafío implicó que la Universidad de Chile se sumara al proceso de formación de docentes y equipos directivos a nivel nacional entre 2015 y 2019.

- En el mismo año, el Ministerio de Educación de El Salvador (Mined) aprobó una reforma a la Ley General de Educación que establece incluir como asignatura obligatoria el estudio de Moral, Urbanidad y Cívica (MUCI), con el fin de fortalecer la capacidad de análisis y discusión para la toma de desiciones, tanto personales como colectivas, que contribuyan al desenvolvimiento en la sociedad de los y las estudiantes. 
En este contexto y en una alianza de colaboración entre la Universidad de Chile y el Mined, surge el proyecto "Intercambio de experiencias pedagógicas entre El Salvador y Chile para fortalecer la formación ciudadana y la convivencia democrática", como estrategia para compartir y difundir las experiencias de formación ciudadana y convivencia escolar que ambas naciones estaban desarrollando.

\section{Implementación del proyecto}

A partir de los contextos descritos, se diseñó un proyecto de colaboración cuyo objetivo general fue contribuir al proceso de capacitación de especialistas salvadoreños en las áreas de formación ciudadana. Para ello se definieron tres líneas de objetivos específicos que derivaron en tres líneas de acción:

- Fortalecer el desarrollo de capacidades en el ámbito de la formación ciudadana y convivencia, a través de asistencia técnica y de intercambio de experiencias y metodologías pedagógicas innovadoras.

- Transferir tecnologías apropiadas para la construcción de una plataforma educativa digital que permita replicar las metodologías, herramientas y experiencias.

- Dejar mecanismos de intercambio de conocimiento instalados entre los actores relevantes.

Las líneas de acción del proyecto buscaron generar procesos sistemáticos y de transformación en el contexto educativo salvadoreño, que promovieran tanto el desarrollo de capacidades docentes en formación ciudadana como el fortalecimiento de equipos técnicos de educación a distancia a nivel ministerial de El Salvador. Estas líneas de acción se organizaron en tres grandes áreas: 


\section{Figura 1}

Líneas de acción del proyecto.

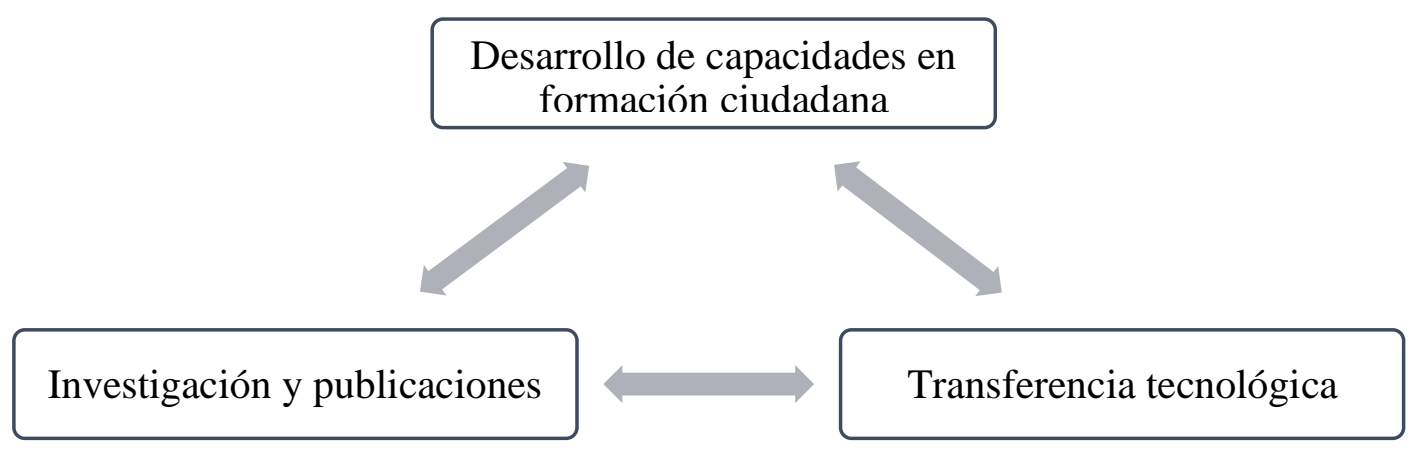

En este artículo se profundizará en los componentes vinculados al desarrollo profesional docente desde la educación a distancia, sin embargo, se enunciarán los demás dispositivos con el fin de proyectar la envergadura del proyecto en su conjunto.

\subsection{Desarrollo de capacidades en formación ciudadana}

Esta línea de acción se proyectó en el diseño de un plan de formación para 70 docentes ${ }^{5}$ especialistas en Ciencias Sociales de El Salvador, con el fin de propiciar un espacio de aprendizaje en modalidad blended learning con foco en la formación ciudadana, cuestión que se tradujo en el diploma "Educación y democracia. Formación ciudadana para la escuela de hoy”, con una extensión de 216 horas cronológicas.

La estructura del programa de formación contemplaba el desarrollo de siete módulos de aprendizaje articulados entre sí, algunos en modalidad completamente virtual, mientras que otros con espacios presenciales, lo que significó un desafío para el diseño instruccional del diploma. Los detalles se observan en la siguiente tabla.

\footnotetext{
${ }^{5}$ Cabe señalar que los y las participantes de esta experiencia de formación se desempeñaban en diversos roles del sistema educativo salvadoreño. En su mayoría eran docentes de centros educativos, sin embargo, también se registraron docentes universitarios, junto con profesionales del Ministerio de Educación de El Salvador.
} 


\section{Tabla 1}

Módulos y modalidad

\begin{tabular}{ll}
\hline Módulos & Modalidad \\
\hline M1: Ciudadanía, convivencia, organización y cultura escolar & B-learning \\
M2: Juventud y ciudadanía & E-learning \\
M3: Convivencia, inclusión y enfoque de derechos & E-learning \\
M4: Experiencias para la innovación en formación ciudadana & E-learning \\
Chile y El Salvador & \\
M5: Formación ciudadana, currículum y pedagogía & B-learning \\
M6: Bases para la formación de formadores & B-learning \\
M7: Sistematización de los aprendizajes & Presencial \\
\hline
\end{tabular}

El diseño instruccional del diploma supuso desde un inicio un doble desafío. El primero vinculado al diseño pedagógico en sí mismo, mientras que el segundo estuvo relacionado con el plano de la contextualización de las temáticas abordadas.

\section{a) DESAFÍO No 1 . DISEÑO DEL DIPLOMA.}

El diseño general se planteó desde tres pilares pedagógicos fundamentales, que se desprenden de la comprensión del aprendizaje profesional situado que permite la reflexión desde la propia práctica. Por ello cada módulo se articuló desde la siguiente tríada:

- Problematización: espacio donde estudiantes, guiados por tutoras, problematizaban desde sus propios contextos y roles los tópicos propuestos por el módulo.

- Profundización: presentación de contenidos centrales a partir de revisiones de literatura que enmarcaran la discusión y, a la vez, ampliaran la mirada de los/as participantes. 
- Generación de acciones: momento en el que, a partir de la problematización y profundización, se propone el diseño de acciones específicas, en base al rol y contexto de cada participante, que permitan abordar los tópicos centrales del módulo.

Asimismo, la propuesta general consideraba una serie de hitos pedagógicos que articulaban el diseño en su conjunto, por ello el diploma contaba con seminarios de profundización, pasantía de participantes salvadoreños en Santiago de Chile y sesiones presenciales que permitieron la generación de vínculos y desarrollo de relaciones de colaboración entre estudiantes.

Tanto los módulos completamente e-learning como los de carácter b-learning contaron con un diseño instruccional que contemplaba videoclases de docentes especialistas en los tópicos centrales; entrevistas académicas y/o con actores del sistema educativo; foros asincrónicos que problematizaban los conceptos del módulos; activadores de aprendizaje, bajo la forma de documentos que recogían un marco teórico de base para la distinción conceptual; talleres de evaluación, consistentes en actividades situadas en el contexto de cada participante, que permitieran la aplicación de los temas abordados; y finalmente, material bibliográfico imprescindible y complementario.

De este modo, el diseño instruccional permitió el desarrollo de los pilares pedagógicos del diploma:

\section{Figura 2}

Pilares pedagógicos y recursos del diploma

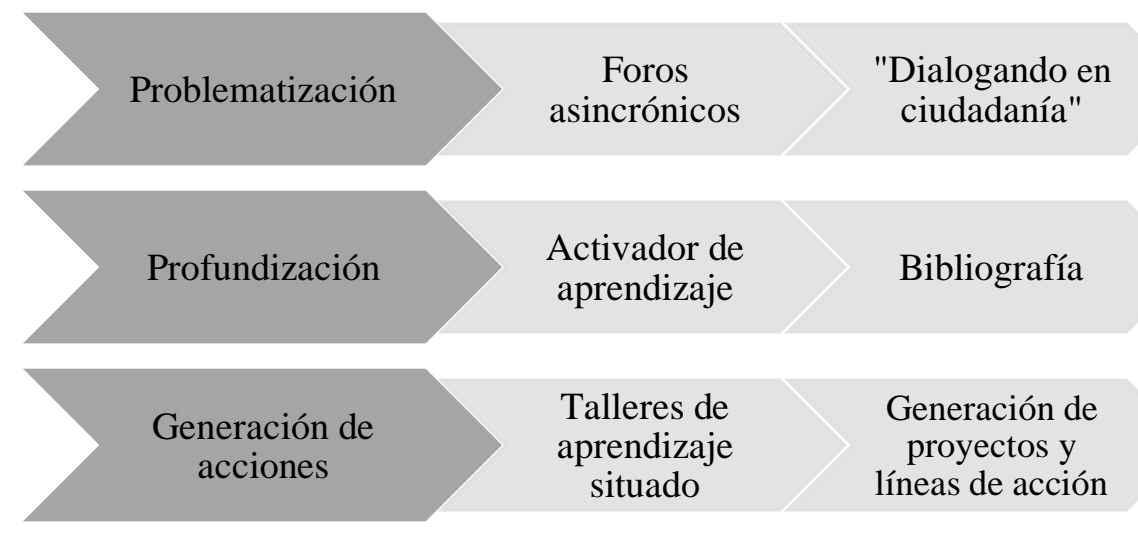


REVISTA SABERES EDUCATIVOS, Nº 5, JULIO-DICIEMBRE 2020

Finalmente, el módulo 7 del diploma propuso el desarrollo de un proyecto articulado a partir de las reflexiones y productos de los módulos anteriores, que permitió una propuesta de fortalecimiento a la formación ciudadana en el contexto salvadoreño.

\section{b) DESAFÍO Nº 2. CONTEXTUALIZACIÓN DEL DIPLOMA}

Por otra parte, y no menos importante, se planteaba el desafío de situar los tópicos generales del diploma en el contexto educativo salvadoreño, lo que fue abordado a través de dos líneas de acción articuladas.

La primera de estas fue la instalación de un proceso de validación de la propuesta de contenidos centrales del diploma y sus módulos, con actores claves del sistema educativo salvadoreño y el Ministerio de Educación. Ello derivó en la inclusión de un módulo de "Juventud y ciudadanía" que problematizaba la construcción de niñez y juventud desde la escuela y sus implicancias para la formación ciudadana, junto con el módulo "Bases para la formación de formadores" que, justamente, permitía que docentes participantes pudiesen replicar la experiencia a escalas regionales.

En cuanto a la segunda línea de acción, esta comprendió un proceso permanente de ajustes por parte del equipo académico y la coordinación del diploma, en articulación con el desarrollo de un plan tutorial específico y abierto a nuevas precisiones identificadas en la propia ejecución, con el fin de mejorar los aspectos pedagógicos y logísticos de la acción formativa, generando al mismo tiempo procesos de reflexión y aprendizaje al interior del propio equipo.

\subsection{Transferencia tecnológica}

En relación a la transferencia tecnológica, el proyecto contempló dos líneas de acción. La primera estuvo centrada en transferir el diseño del diploma a servidores del Ministerio de Educación para que, como unidad de educación a distancia, contaran con el modelo formativo para posibles ejecuciones posteriores que enriquecieran la educación continua de docentes 
en el ámbito de la formación ciudadana, proceso que se fue desarrollando a lo largo del proyecto.

La segunda línea de acción que se propuso fue precisamente que, a partir de la tarea de transferencia tecnológica, se fortalecieran las capacidades en equipos técnicos salvadoreños, a través de talleres de formación y la generación de material pedagógico que permitiera la replicabilidad de la experiencia desde la educación a distancia.

Estos objetivos plantearon dos desafíos a la transferencia tecnológica:

\section{a) DESAFÍO N³. CONFORMACIÓN DE EQUIPOS}

El desarrollo de la transferencia tecnológica que implicó el proyecto supuso un ejercicio inicial de trabajo con un equipo espejo, vale decir, que presentara capacidades y roles similares en tanto equipo técnico de educación a distancia (encargado/a de plataforma, diseñador/a instruccional, productor/a audiovisual, especialista en informática), tanto para el equipo de la Universidad de Chile como para el del Ministerio de Educación de El Salvador.

Este proceso inicial se desarrolló a partir de variados niveles de flexibilidad, ya que no fue posible encontrar esta condición de entrada y más bien fue desarrollada en el transcurso del proyecto, contando al finalizar con un equipo de profesionales salvadoreños con los perfiles requeridos para esta tarea.

\section{b) DESAFÍO No 4. FORTALECIMIENTO DE EQUIPOS TÉCNICOS}

Este proceso se desarrolló a partir de tres jornadas de formación de carácter presencial, en las que se abordaron temas específicos y estratégicos de la educación a distancia para docentes.

- La primera jornada abordó temáticas transversales como arquitectura general, métodos de instalación, configuración, personalización y administración de plataforma. 
- La segunda jornada profundizó en el diseño instruccional, en el contexto de la cultura y la educación digital, y en la producción audiovisual como generadora de objetos de aprendizaje (principios y técnicas).

- La tercera jornada abordó la revisión de los componentes digitales del diploma, sobre la base del diseño instruccional del mismo: simulación de construcción y montaje de un módulo $100 \%$ digital, y administración y recursos para la dictación y replicabilidad del diploma.

\subsection{Investigación y publicaciones}

Esta línea, si bien se configuró desde el inicio del proyecto, fue abriendo nuevos caminos para su abordaje desde las necesidades del propio proyecto y el contexto educativo salvadoreño.

Cabe destacar aquí dos desafíos fundamentales que se abordaron desde el proyecto. El primero estuvo vinculado a la colaboración de actores del sistema educativo salvadoreño, mientras que el segundo estuvo relacionado con la generación de material bibliográfico desde las acciones del mismo proyecto.

\section{a) DESAFÍO No 5. INCORPORACIÓN DE VOCES DE ACTORES DEL CONTEXTO EDUCATIVO SALVADOREÑO}

Uno de los elementos de base para la comprensión de la formación ciudadana y el aprendizaje está vinculado con relevar la voz de quienes son parte de los procesos. Al respecto, en este programa se relevó la percepción de los actores en distintas instancias de generación de conocimiento y difusión de este.

Es así como se generaron seminarios abiertos con el fin de intercambiar experiencias, donde los/as protagonistas fueron algunos/as de los/as estudiantes, destacando su participación tanto en seminarios desarrollados en Santiago como en San Salvador. Asimismo, se publicaron artículos sobre la experiencia de formación a través de la pasantía en Santiago de algunos participantes del diploma. 
Finalmente, cabe destacar el desarrollo de dos procesos de investigación que dieron cuenta de la voz de actores. Uno de ellos estuvo centrado en el levantamiento de la experiencia de formación, tres meses después de la finalización del programa. Asimismo, se levantó un estudio sobre la percepción de docentes a nivel nacional en El Salvador, sobre la implementación de la asignatura de "Moral, Urbanidad y Cívica (MUCI)".

En forma especial, es necesario relevar el estudio sobre la implementación del diploma, ya que recogió información fundamental sobre su pertinencia y sus aportes al desarrollo profesional docente en base a las siguientes dimensiones:

- Desarrollo profesional docente en la acción formativa.

- Pertinencia al contexto.

- Entrega de contenidos y desarrollo de reflexiones en torno al rol de formador de formadores.

- Situación de los proyectos finales en la escuela.

Los resultados arrojan que entre un $60 \%$ y $80 \%$ los/as participantes considera que los contenidos y reflexiones fueron útiles, al tiempo que pertinentes y flexibles a las necesidades del contexto. Centrando la atención en especial en la dimensión "desarrollo profesional”, se puede señalar que más de un $80 \%$ reconoce que el diploma favoreció las reflexiones profesionales, individuales y colectivas, en torno a la formación ciudadana en la escuela y a sus propias prácticas ${ }^{6}$, estructura de base para el aprendizaje profesional.

\section{b) DESAFÍO Nº 6 . GENERAR MATERIAL BIBLIOGRÁFICO}

Finalmente, el proyecto se propuso el desarrollo de material bibliográfico que recogiera los aprendizajes de la experiencia del diploma. En esta línea es que se publicaron dos libros con artículos que se desprenden de los módulos del proceso formativo y de la pasantía desarrollada:

\footnotetext{
${ }^{6}$ Fuente: Informe final de seguimiento ex post, diploma "Educación y democracia: formación ciudadana para la escuela de hoy" (documento no accesible públicamente).
} 
- Educar para la ciudadanía: fundamentos, metodologías y desarrollo profesional docente. Álvaro Ramis y Marcela Peña, compiladores.

- Educación y democracia: formación ciudadana para la escuela de hoy. Álvaro Ramis y Carlos Rodríguez, compiladores.

Estas publicaciones hoy son parte de la bibliografía del mismo diploma y otros procesos de formación que desarrolla tanto el Ministerio de Educación de El Salvador como Saberes Docentes.

\section{Logros del proyecto}

\subsection{Resultados de satisfacción}

En este punto es importante identificar algunos resultados, en especial sobre la implementación del diploma. Estos corresponden al análisis de encuestas aplicadas al total de los y las participantes, a partir de diversos ítems que se resumen en la siguiente tabla:

\section{Tabla 2}

Resultados de encuesta satisfacción del diploma

\begin{tabular}{lcccccc}
\hline Ámbito & Excelente & Bueno & Total & Regular & Malo & Total \\
\hline Objetivos y contenidos & $93,1 \%$ & $6,9 \%$ & $100,0 \%$ & $0,0 \%$ & $0,0 \%$ & $0,0 \%$ \\
Actividades pedagógicas & $89,4 \%$ & $7,6 \%$ & $97,1 \%$ & $0,0 \%$ & $0,0 \%$ & $0,0 \%$ \\
Plataforma virtual & $85,0 \%$ & $2,1 \%$ & $87,1 \%$ & $0,7 \%$ & $0,0 \%$ & $0,7 \%$ \\
\hline
\end{tabular}

El ámbito con mayor porcentaje de logro fue "Objetivos y contenidos" (evaluado como “excelente" por el 93,1\%), lo que tiene relación con la consistencia de los objetivos declarados y los contenidos desarrollados, el cumplimiento de estos y la coherencia en la 
organización de los módulos. En los comentarios realizados por los/as participantes destacó principalmente la metodología empleada y los recursos de aprendizaje. Respecto de lo que se podría mejorar, se sugirió ajustar los tiempos y dar más flexibilidad en la entrega de tareas.

Algunos comentarios de los/as participantes destacan tanto las metodologías como las temáticas abordadas, lo que se puede apreciar en algunos comentarios: "Material innovador. Metodologías participativas e interactivas. Criterios de evaluación preestablecidos”, "Considero que la temática abordada es pertinente a la realidad educativa que actualmente poseemos", "El material complementario es muy bueno y adecuado a los contenidos. Los profesores son excelentes y el enfoque del diploma satisface la demanda nacional”.

Como se puede observar, la implementación del plan de formación cumplió las expectativas de los/as docentes participantes, destacando, precisamente, las actividades pedagógicas, junto con los objetivos y contenidos propuestos.

\subsection{Metas logradas del proyecto}

El proyecto se propuso una serie de metas en base a los componentes ya señalados. A continuación, se destacan las más relevantes: 


\section{Tabla 3}

Metas y logros del proyecto ${ }^{7}$

\begin{tabular}{|c|c|c|}
\hline $\begin{array}{l}\text { Línea de } \\
\text { acción }\end{array}$ & Metas propuestas & Logro \\
\hline \multirow{4}{*}{$\begin{array}{l}\text { Desarrollo de } \\
\text { capacidades }\end{array}$} & $\begin{array}{l}70 \text { docentes del núcleo de especialistas participan } \\
\text { en proceso formativo de modalidad semipresencial } \\
\text { "Diploma educación y democracia. Formación } \\
\text { ciudadana para la escuela de hoy". }\end{array}$ & $\begin{array}{l}\text { Logrado: } 74 \\
\text { docentes } \\
\text { participaron en el } \\
\text { proceso de } \\
\text { formación. }\end{array}$ \\
\hline & $\begin{array}{l}8 \text { docentes salvadoreños y } 2 \text { profesionales del } \\
\text { Ministerio de Educación participan en la pasantía en } \\
\text { Chile "Colaboración conjunta en experiencias } \\
\text { educativas en el ámbito de la formación ciudadana } \\
\text { y la convivencia". }\end{array}$ & $\begin{array}{l}\text { Logrado: se } \\
\text { desarrolló la } \\
\text { pasantía con } 10 \\
\text { profesionales } \\
\text { salvadoreños. }\end{array}$ \\
\hline & $\begin{array}{l}55 \text { docentes obtienen al menos un } 70 \% \text { en la } \\
\text { evaluación de habilidades y conocimientos del } \\
\text { diploma. }\end{array}$ & $\begin{array}{l}\text { Logrado: } 58 \\
\text { docentes se } \\
\text { certifican. }\end{array}$ \\
\hline & $\begin{array}{l}1 \text { libro metodológico editado y publicado para el } \\
\text { fortalecimiento de la formación ciudadana en la } \\
\text { escuela. }\end{array}$ & $\begin{array}{l}\text { Logrado: edición y } \\
\text { lanzamiento de dos } \\
\text { libros }\end{array}$ \\
\hline \multirow[t]{4}{*}{$\begin{array}{l}\text { Transferencia } \\
\text { tecnológica }\end{array}$} & $\begin{array}{l}4 \text { módulos a distancia desarrollados e } \\
\text { implementados en la plataforma EduAbierta. }\end{array}$ & $\begin{array}{l}\text { Logrado: se } \\
\text { desarrollaron } 7 \\
\text { módulos e-learning }\end{array}$ \\
\hline & 1 versión $100 \%$ a distancia del diploma & Logrado \\
\hline & 1 manual de transferencia tecnológica elaborado. & Logrado \\
\hline & $\begin{array}{l}7 \text { profesionales salvadoreños capacitados/as en las } \\
\text { metodologías y tecnologías para el desarrollo de } \\
\text { plataformas de educación a distancia. }\end{array}$ & $\begin{array}{l}\text { Logrado: equipo de } \\
13 \text { profesionales } \\
\text { fue formado en } \\
\text { educación digital. }\end{array}$ \\
\hline
\end{tabular}

\footnotetext{
${ }^{7}$ Síntesis elaborada en base a referencias publicadas en la memoria del proyecto (Saberes Docentes, 2019).
} 
Finalmente, cabe señalar que durante el año 2020 se implementó una segunda versión del diploma, bajo la completa responsabilidad del Ministerio de Educación de El Salvador, donde el rol de tutoría fue desarrollado por destacados/as participantes de la primera versión impartida por la Universidad de Chile.

\section{Desafíos para la educación continua de docentes y otros actores del sistema educativo}

El diseño e implementación del proyecto "Intercambio de experiencias pedagógicas entre El Salvador y Chile para fortalecer la formación ciudadana y la convivencia democrática" genera una serie de reflexiones en torno a la educación continua y sus desafíos, en especial sobre el papel que juega la modalidad a distancia como parte fundamental de este proyecto, no solo en cuanto a la ejecución del diploma, sino también desde su componente de transferencia tecnológica.

Antes que nada, desde la educación continua, es necesario profundizar en lo que se refiere a los diseños pedagógicos, específicamente aquellos orientados a docentes en modalidad $e$ learning. La propuesta del diploma y otros espacios de formación del proyecto se basaron en principios de aprendizaje experiencial (Kolb, 1981), los que proponen una reflexión situada desde cada una de las temáticas abordadas y del plan de formación en su conjunto.

En este sentido, como punto inicial para el aprendizaje, es prioritario relevar el conocimiento y experiencia de los y las participantes, a través de espacios para compartir prácticas y, sobre todo, para la búsqueda de reflexiones pedagógicas en base a situaciones reales, a través de actividades auténticas desarrolladas en forma colectiva. En este proyecto esta estrategia fue fundamental, ya que el conocimiento del contexto educativo salvadoreño lo aportaban los y las estudiantes. En cuanto a esto, surgen algunas interrogantes fundamentales.

¿Qué rol juegan los equipos tutoriales? Es imposible pensar el diseño de una propuesta de formación a distancia sin problematizar los roles y perfiles de quienes desarrollan la tutoría 
en entornos virtuales de aprendizaje, desafiando un modelo lineal de entrega de conocimiento para transitar a un enfoque que gatille las interacciones, a través de recursos sincrónicos y asincrónicos. En este sentido, el/la tutor/a debe "estar preparado para generar un diálogo efectivo con los participantes y entre los participantes, que favorezca el aprendizaje activo, la construcción de conocimiento cooperativo y/o colaborativo" (Gros y Silva, 2005. p.4).

Salmon (2000) señala que existen cinco etapas en el proceso de mediación tutorial: acceso y motivación, socialización, compartir información, construcción de conocimiento y desarrollo. El desafío es generar estos procesos con un perfil de estudiantes (en este caso docentes) que no están familiarizados/as con las herramientas e interacciones a través de plataformas virtuales y que, desde su propia práctica, valoran el vínculo presencial en el aula como medio de aprendizaje.

Desde este prisma, la experiencia presentada logró establecer interacciones y relevar los saberes de los/as estudiantes a través de dos instancias clave. La primera es la propuesta de problematización, tanto en foros como en actividades de formación centradas en la experiencia de docentes en formación ciudadana, lo que permitió establecer un diálogo entre pares en forma cercana y reflexiva. La segunda instancia es la publicación y presentación de sus trabajos en espacios como seminarios y boletines, que posicionaran la voz de los actores y sus contextos, aspecto que generó gran motivación en los y las participantes.

¿Cuál es el rol de los equipos académicos en estas experiencias de aprendizaje? Estos generan una serie de materiales y reflexiones desde la literatura, proponiendo perspectivas actualizadas y apoyadas en la investigación, que permiten mediar la búsqueda de soluciones y propuestas a las problemáticas planteadas por estudiantes. Sin embargo, es importante también identificar como elemento clave el desarrollo de competencias pedagógicas que permitan proyectar el diseño de recursos de aprendizaje con sentido.

Desde esta perspectiva, si bien es cierto que este trabajo es apoyado por la figura de el/la diseñador/a instruccional como profesional fundamental para el logro de objetivos de aprendizaje, el aporte de académicos/as con un enfoque pedagógico, en especial de educación continua, es clave para la contextualización de diseños y ajustes de los materiales instruccionales. En el proyecto la mayoría de los/as académicos/as estaban vinculados/as a 
DESARROLLO PROFESIONAL DOCENTE Y EDUCACIÓN A DISTANCIA. UNA EXPERIENCIA DESDE LA COOPERACIÓN SUR-SUR / PEÑA-RUZ

procesos de formación continua de docentes, en especial la coordinación académica del programa, lo que permitió orientar con sentido el diseño de cada propuesta.

¿De qué forma los recursos pedagógicos median el aprendizaje experiencial? Los materiales y recursos diseñados deberían responder a la necesidad de transformar los contextos educativos desde una reflexión problematizadora, una indagación teórica que permita proponer estrategias y acciones que aporten al cambio de las prácticas docentes, en forma contextualizada y pertinente. De este modo, el diseño del diploma logró proponer tareas auténticas (Monereo, 2003), vale decir, vinculadas al desempeño de los y las participantes, generando sinergia en el aprendizaje situado.

¿Cuál es el aporte de la formación continua al desarrollo profesional docente? La respuesta a esta pregunta es fundamental para identificar en qué medida la experiencia de formación vivida genera cambios en las prácticas profesionales y, con ello, proponer ajustes a los procesos formativos en virtud del desarrollo profesional. El proyecto presentado generó un proceso de levantamiento de información posterior a la ejecución, en distintas dimensiones, donde se identificó que los y las participantes valoraron la revisión bibliográfica y las actividades pedagógicas desarrolladas, reconociendo que aportan a la comprensión de ciertos fenómenos educativos y a la reflexión, tanto individual como colectiva, sobre los mismos. Sin embargo, los datos muestran que un $26 \%$ no logró implementar el proyecto de acción para el fortalecimiento de la formación ciudadana diseñado, mientras que un $56 \%$ señala que logró implementar solo algunos componentes, argumentando diversas razones como contextos, recursos y apoyos de otros actores educativos.

¿Cuál es el rol de los equipos técnicos y pedagógicos en educación a distancia? Otro aspecto que desafía la educación continua tiene que ver con problematizar las características y perfiles de los equipos técnicos de educación a distancia, ya que estos juegan un papel fundamental en la implementación de las propuestas pedagógicas. Se incluye aquí a profesionales del ámbito audiovisual, del diseño gráfico e instruccional, quienes desde su experticia generan una perspectiva que marca una trayectoria pedagógica de las acciones formativas. En este sentido, se visualiza como estratégico contar con experiencia específica en estos roles en educación continua y, particularmente, de actores educativos. Esto permite 
REVISTA SABERES EDUCATIVOS, Nº 5, JULIO-DICIEMBRE 2020

la toma de decisiones con fundamentos sólidos en relación a la elaboración de productos, en el marco de una relación recíproca entre los equipos académicos y técnicos, que refleje un diálogo constructivo que dé sentido a la acción formativa y tenga sentido para los y las participantes.

A modo de síntesis, los principales aprendizajes de la experiencia presentada para la educación continua se pueden organizar en seis puntos claves:

\section{Figura 3}

Puntos clave para el diseño e implementación de programas de educación continua.

1. Diagnósticos que permitan la contextualización de tópicos, sentidos y reflexiones.

2. Colaboración entre pares en todo nivel.

3. Equipos académicos, tutoriales y técnicos con experiencia en educación continua.

4. Diseños pedagógicos que promuevan actividades situadas y auténticas.

5. Generación de saberes desde y con los/as participantes.

6. Seguimiento de aprendizajes en el contexto laboral.

Es posible observar, a través del desarrollo de este proyecto, los múltiples desafíos que se plantean desde la educación continua a distancia y las implicancias que esta tiene para el desarrollo profesional docente. Esto hace que hoy sea una necesidad abordarla desde las universidades y centros de formación, con un enfoque que recoja los saberes de los actores, promueva actividades situadas en los contextos y estimule la reflexión sobre la práctica y transformación de esta.

Es evidente que en el proceso de formación en modalidad e-learning (o b-learning) son variados los actores y perfiles que dan cuerpo a los programas formativos. Por ello es 
DESARROLLO PROFESIONAL DOCENTE Y EDUCACIÓN A DISTANCIA. UNA EXPERIENCIA DESDE

LA COOPERACIÓN SUR-SUR / PEÑA-RUZ

fundamental relevar la experiencia de éstos en el diseño de educación continua para docentes y actores educativos, ya que son quienes entregan un marco de actuación para dichos procesos.

Finalmente, es igualmente importante levantar información a través de estudios que permitan ampliar el conocimiento específico sobre la educación continua docente en las modalidades b-learning o e-learning y su real aporte al desarrollo profesional. Hoy existe una amplia oferta de programas de formación a distancia y, en este contexto, bien vale preguntarse: ¿cuál es el aporte que realizan al aprendizaje de docentes en ejercicio y a su desarrollo profesional?

\section{Referencias}

Duarte, A., Guzmán, F. y Domínguez, Y. (2018). Aportaciones de la formación blended learning al desarrollo profesional docente. RIED: Revista Iberoamericana de Educación a distancia, 21(1), 155-174. doi: https://doi.org/10.5944/ried.21.1.19013

García-Ruiz, R., Aguaded, I., y Bartolomé, A. (2017). La revolución del "blended learning” en la educación a distancia. RIED. Revista Iberoamericana de Educación a Distancia, 21(1), 25-32. doi: http://dx.doi.org/10.5944/ried.21.1.19803

Gros, B., Silva, J. (2005). La formación del profesorado como docente en los espacios virtuales de aprendizaje, Revista iberoamericana de educación, núm.,36 (1). Recuperado de https://rieoei.org/historico/deloslectores/959Gros.PDF

Kolb, D. (1981). Learning styles and disciplinary differences. Recuperado de https://www.researchgate.net/publication/283922529_Learning_Styles_and_Discipl inary_Differences

Korthagen, F., Loughran, J. y Russell, T. (2006). Developing fundamental principles for teacher education programs and practices. Teaching and Teacher Education, 22(8), 1020-1041. 
REVISTA SABERES EDUCATIVOS, Nº 5, JULIO-DICIEMBRE 2020

Laveault, D. (2016.) Chapter 8: Building Capacity: Professional Development and Collaborative Learning About Assessment. En D. Laveault y L. Allal, (Eds.), Assesment for Learning: Meeting the Challenge of Implementation (pp. 131- 143). New York - London: Springer.

Ley 20.911. Diario Oficial de la República de Chile, Santiago, Chile, 28 de marzo de 2016. Recuperado de http://bcn.cl/1v0oy

Ministerio de Educación, Ciencia y Tecnología de El Salvador. (2018). Moral, Urbanidad y Cívica. Programas de Primero y Segundo Ciclo de Educación Básica. Recuperado de

http://www.mined.gob.sv/jdownloads/Nuevos\%20Programas\%20de\%20Estudio/Pr ogramas\%20Moral\%20y\%20C\%C3\%ADvica/Programas\%20de\%20estudio/PROG RAMA\%20I\%20y\%20II\%20BASICA\%20CS6.compressed.pdf

Monereo, C. (2003) La evaluación del conocimiento estratégico a través de tareas auténticas. Pensamiento Educativo 32, 71-89. Recuperado de http://www.pensamientoeducativo.uc.cl/files/journals/2/articles/246/public/246572-1-PB.pdf

Saberes Docentes. (2019). Memoria proyecto Intercambio de experiencias pedagógicas entre El Salvador y Chile para fortalecer la formación ciudadana y convivencia democrática. Recuperado de https://www.fondochile.cl/wpcontent/uploads/2019/02/Memoria-Universidad-de-Chile-El-Salvador-2017-20191.pdf

Salmon, G. (2000). E-moderating: The key to teaching and learning online. London: Kogan Page.

Vaillant, D. (2016). El fortalecimiento del desarrollo profesional docente: una mirada desde Latinoamérica. Journal of Supranational Policies of Education, (5), 5-21. Recuperado de http://dx.doi.org/10.15366/jospoe2016.5 